\title{
HEMATOPOIESIS
}

\section{The Effect of Recombinant Human Erythropoietin on Platelet Counts Is Strongly Modulated by the Adequacy of Iron Supply}

\author{
By Martine Loo and Yves Beguin
}

\begin{abstract}
The effect of recombinant human erythropoietin (rHuEpo) on megakaryopoiesis remains controversial. Treatment with rHuEpo in renal failure patients has been associated with a slight elevation of platelet counts. In animal studies, high doses of rHuEpo produced an increase of platelet counts followed by a gradual retum to nomal after 7 to 15 days or even a substantial degree of thrombocytopenia. However, because iron deficiency is also known to be associated with thrombocytosis, (functional) iron deficiency during rHuEpo could be contributing to these observations. We investigated the impact of iron supply on changes in platelet counts induced by rHuEpo. Rats were either fed normal food (normal rats) or received 1\% carbonyl iron for 2 weeks or 3 months, as well as during the experiment, to achieve iron supplementation or overload, respectively. Rats of all three categories then received daily intravenous injections of rHuEpo (10, 50, or $\mathbf{1 5 0} \mathrm{U}$ ) or normal saline (0 U) for $\mathbf{2 0}$ days. With 0 to $10 \mathrm{U}$ rHuEpo, platelets remained stable. In normal rats receiving $\mathbf{5 0}$ to $\mathbf{1 5 0} \mathbf{U}$ rHuEpo, platelets increased to $120 \%$ to $140 \%$ of baseline at 4 to 12 days to level off at $120 \%$ at $\mathbf{1 6}$ to 20 days. This response was less sustained in splenectomized animals. Iron-supplemented rats receiving 50 to $150 \mathrm{U}$ rHuEpo also increased platelets initially, but the
\end{abstract}

$\mathbf{E}$ RYTHROPOIETIN (Epo) is the primary erythropoietic growth factor, but it has also been shown to affect platelet production. In large clinical trials of recombinant human Epo (rHuEpo) in renal failure patients, platelet counts increased significantly within 2 weeks and then gradually returned to pretreatment levels after months of maintenance therapy.,2 Mean platelet volumes decreased, but platelet counts remained unchanged in patients receiving rHuEpo and oral iron before scheduled surgery. ${ }^{3}$ In adult but not in infant monkeys, rHuEpo therapy resulted in elevated platelet counts throughout the 6-week treatment, followed by rapid normalization thereafter. ${ }^{4}$ In dogs, mice, or rats, short-term treatment with high doses of rHuEpo has been shown to stimulate platelet production, but platelet counts tended to normalize after 7 to 15 days. ${ }^{5-9}$

From the Department of Medicine, Division of Hematology, University of Liège, Liège, Belgium.

Submitted October 30, 1998; accepted December 9, 1998.

Supported in part by Grants No. 3.4555.91, 3.4621.94, and 7.4538.96 from the FNRS. M.L. was supported by a grant from "Télévie" (FNRS, Belgium) and by a grant from the "Fondation Frédéricq" (University of Liège, Belgium). Y.B. is a Senior Research Associate of the National Fund for Scientific Research (FNRS, Belgium).

Address reprint requests to Yves Beguin, MD, University of Liège, Department of Hematology, CHU Sart-Tilman, 4000 Liège, Belgium; e-mail: yves.beguin@chu.ulg.ac.be.

The publication costs of this article were defrayed in part by page charge payment. This article must therefore be hereby marked "advertisement" in accordance with 18 U.S.C. section 1734 solely to indicate this fact.

(1) 1999 by The American Society of Hematology.

0006-4971/99/9310-0030\$3.00/0 peak was at day 4, followed by a gradual retum to baseline and even a moderate thrombocytopenia later on. Ironoverloaded rats receiving 50 to $\mathbf{1 5 0} \mathrm{U}$ rHuEpo also had increased platelets at day 4, but the duration of platelet increase was shorter, and they experienced a more pronounced degree of thrombocytopenia in proportion to the dose of rHuEpo. Because the early elevation of platelets was of larger magnitude than hematocrit changes, it is unlikely that it could be accounted for by shrinkage of plasma volume. Because it was observed in all three iron conditions, there appears to be some direct positive effect of rHuEpo on platelet production. However, after this transient effect, expanded erythropoiesis appears to exert a negative impact upon platelet production. Secondary thrombocytopenia was not related to splenic pooling, and its very slow correction after cessation of rHuEpo therapy is not compatible with changes in platelet survival. Rather, it is consistent with stem cell competition between erythroid and megakaryocytic development. However, this secondary thrombocytopenia is masked by (functional) iron deficiency in rats not receiving an adequate iron supply from food or stores.

(1) 1999 by The American Society of Hematology.

However, transgenic mice expressing the human Epo gene develop both polycythemia and a moderate degree of thrombocytopenia. ${ }^{10}$ Actually, large, chronic doses of rHuEpo also caused thrombocytopenia in mice, and stem cell competition between erythroid and platelet precursors has been suggested as the cause of this phenomenon. ${ }^{11}$

In a previous study of patients with end-stage renal disease, we demonstrated that platelet changes in response to rHuEpo therapy correlated with modifications of erythropoietic activity. ${ }^{2}$ However, enhanced erythropoiesis is also associated with modifications of iron metabolism. In particular, functional iron deficiency, ie, an imbalance between iron needs in the bone marrow and iron supply from stores, may develop even in the presence of adequate storage iron when these stores cannot be mobilized rapidly enough. ${ }^{2}$ Iron deficiency has been shown to be associated with reactive thrombocytosis. ${ }^{13-17}$ Iron supplementation in iron-deficient infants ${ }^{18}$ or rats ${ }^{19,20}$ was rapidly followed by a return of platelet counts to normal levels.

Therefore, we undertook the present study to investigate the impact of iron supply on changes in platelet counts induced by rHuEpo. We studied the effect of various doses of rHuEpo and various durations of treatment on both erythropoiesis and megakaryopoiesis. We also examined the potential role of the spleen in this setting, because some studies had suggested that splenic pooling could interfere with these observations. ${ }^{7,21,22} \mathrm{We}$ observed a diphasic pattern of response of platelet counts to rHuEpo, with the initial increment of platelet counts being followed by substantial thrombocytopenia. The study demonstrated that the iron status of the animals played an important role in the second phase of the diphasic response, with iron deficiency protecting them from thrombocytopenia. 
A

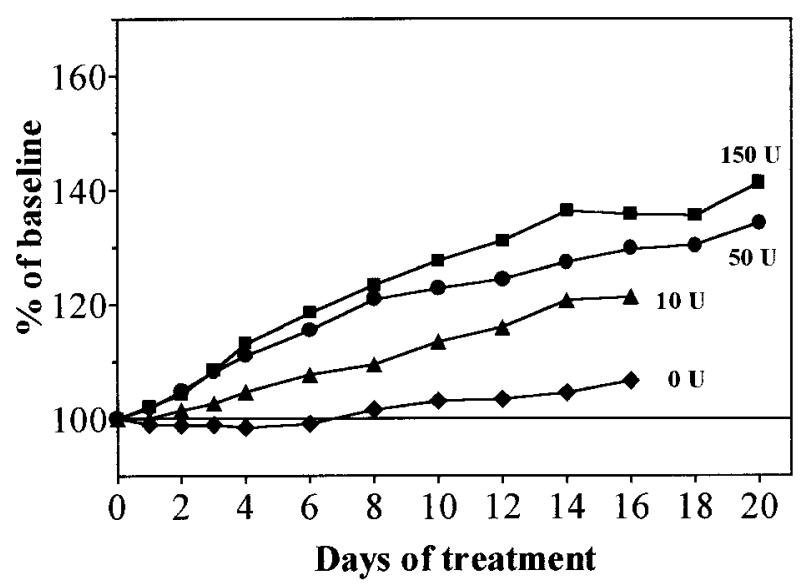

$\mathbf{B}$

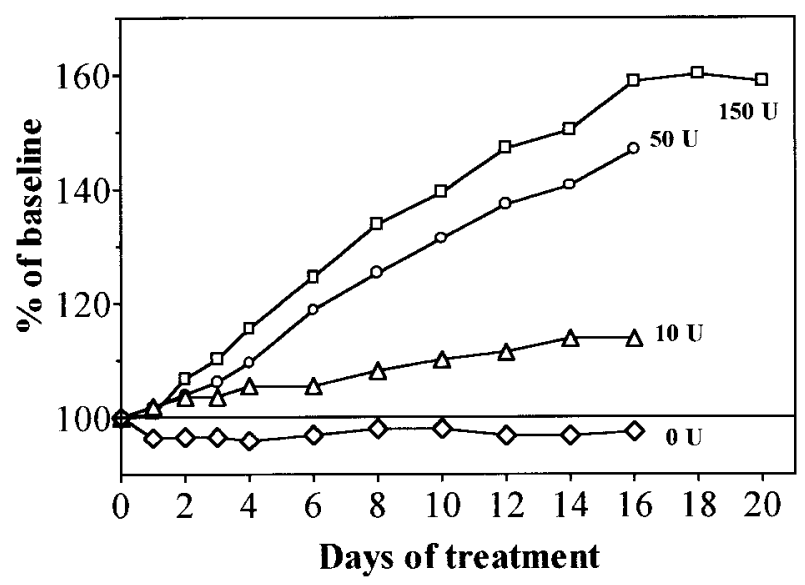

Fig 1. Evolution of Hct during treatment with daily doses of 0 (diamonds), 10 (triangles), 50 (circles), or 150 (squares) U rHuEpo. Results are expressed as percentages of baseline value. (A) Normal rats (solid symbols); (B) overloaded rats (open symbols).

\section{MATERIALS AND METHODS}

Animals. Male Wistar rats were obtained from Janssen (Beerse, Belgium) and raised at the University of Liège (Liège, Belgium) from the age of 3 weeks through the end of the experiment. One group of animals (normal rats) was fed standard granular food. Other groups of rats were fed standard food in powder form supplemented with $1 \%$ (wt/wt) carbonyl iron (Sigma Chemical Co, St Louis, MO) 6 days per week and normal granular food 1 day per week, for a total of 2 weeks (supplemented rats) or 3 months (overloaded rats) before as well as during the experiment, to achieve iron supplementation or iron overload, respectively. Some animals underwent splenectomy under general anesthesia with ether (Gifer Barbezat, Dicenes, France) followed by placement of Autoclip Mikron clips (Becton Dickinson Benelux, Erembodegem-Alst, Belgium) for 10 days. They were then left to stabilize for 30 days before the experiment, and only animals showing no sign of inflammation at that time were used. Other rats underwent the same procedure but without splenectomy (sham-operated).
Epo therapy. rHuEpo (Recormon) was kindly provided by Boehringer Mannheim (Brussels, Belgium). Rats were injected daily with intravenous rHuEpo at a dose of 10,50 , or $150 \mathrm{U} / \mathrm{d}$ for up to 20 days. Control animals ( $0 \mathrm{U}$ rHuEpo) received the same volume of normal saline.

Laboratory analyses. Blood samples were drawn from a tail vein under short ether anesthesia 3 times per week just before rHuEpo injections. Part of the blood was drawn on ACD formula A and part on heparin to be centrifuged to obtain plasma, which was frozen at $-20^{\circ} \mathrm{C}$ until processing. Complete blood counts were measured on a Technicon H1 automatic cell counter (Technicon, Tarrytown, NY), with appropriate corrections for dilution by ACD. Preliminary experiments showed that a minor proportion of the smaller rat platelets could not be detected by the counter. However, this fraction was constant from rat to rat as well as over time and platelet volume did not change during rHuEpo therapy. Expressing the results as the percentage of baseline values thus eliminated this problem. The small fraction of larger platelets was

A
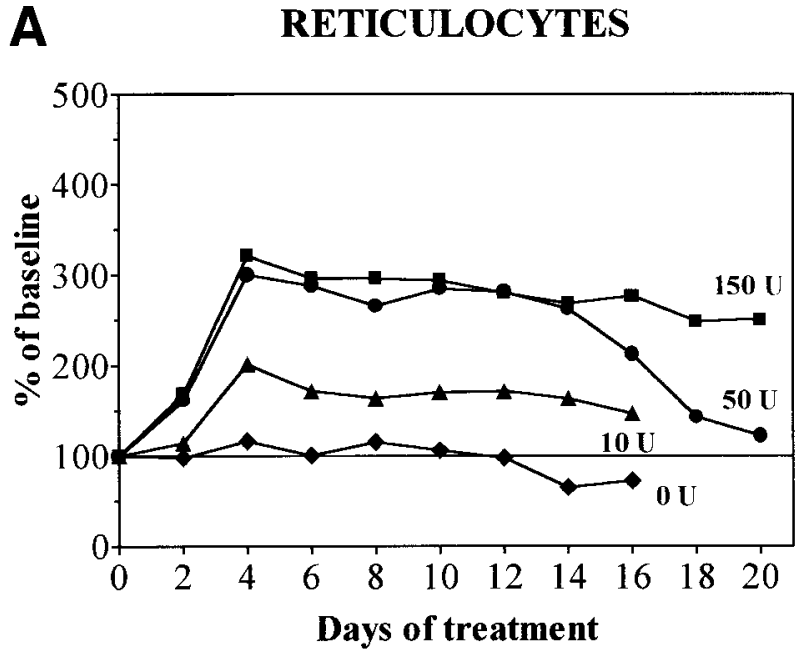

B RETICULOCYTES

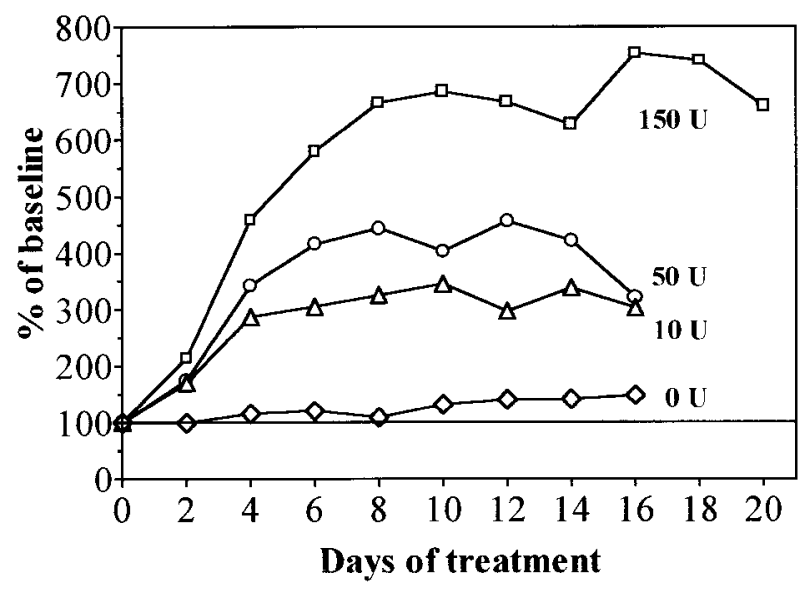

Fig 2. Evolution of absolute reticulocyte count during treatment with daily doses of $\mathbf{0}$ (diamonds), 10 (triangles), 50 (circles), or 150 (squares) U rHuEpo. Results are expressed as percentages of baseline value. (A) Normal rats (solid symbols); (B) overloaded rats (open symbols). 
appropriately detected. The percentage of reticulocytes was determined by cytofluorometry on a FACSCAN cytofluorometer (Becton Dickinson, San Jose, CA) after coloration with thiazole orange. ${ }^{23}$ Preliminary experiments showed that this automated method gave results similar to manual counting on blood smears colored with brilliant cresyl blue. Plasma soluble transferrin receptor (sTfr) was measured as previously described with minor modifications. ${ }^{24,25}$ After varying durations of treatment, a subset of the animals were exsanguinated from the abdominal aorta and perfused with 20 to $30 \mathrm{~mL}$ saline solution. The spleen was removed and weighted. Nonheme iron was extracted from the liver, ${ }^{26}$ and iron concentration was determined by proton-induced x-ray emission. ${ }^{27}$ Serum iron (SeFe) and total iron binding capacity (TIBC) were measured using standard methods, ${ }^{28}$ and transferrin saturation was derived from these figures.

Statistical methods. All results were expressed as percentages of a baseline value and expressed as the mean \pm standard deviation $(\mathrm{M} \pm$ $\mathrm{SD})$. Comparisons of baseline values with later measurements in the same group of animals were performed using paired Student's $t$-tests. Comparisons between groups were performed with Student's $t$-tests, with Welsch's correction in case of unequal variances. Most statistical analyses were performed with the Excel 97 (Microsoft Corp, Redmond, WA) or Prism 2.0 (GraphPad Software Inc, San Diego, CA) software.

\section{RESULTS}

During treatment with $\mathrm{rHuEpo}$, a progressive erythroid response was observed and its amplitude was similar in unmanipulated, sham-operated, or splenectomized animals. This response was proportional to the dose of rHuEpo (Figs 1 and 2). Low doses (10 U) of rHuEpo produced some increment of hematocrit (Hct; Fig 1) and reticulocytes (Fig 2) without any change in sTfR levels, suggesting that this was achieved through shift reticulocytosis rather than true stimulation of erythropoiesis. Higher doses caused more substantial increases of sTfR, reticulocytes, and Hct. The iron status of the animals strongly modulated the erythroid response, being significantly more prominent in overloaded rats compared with supplemented and normal rats, even if the initial slope of Hct increase was similar in overloaded compared with normal rats (Fig 3). After 20 days of treatment with $150 \mathrm{U}$ rHuEpo, the Hct of overloaded rats attained a plateau at $160 \%$ of baseline values, whereas normal rats only achieved a $135 \%$ plateau (Fig 1). Reticulocytes attained levels 7 times versus 3 times baseline in overloaded and normal animals, respectively (Fig 2).

Spleen weight remained stable with 0 or $10 \mathrm{U}$ rHuEpo, but increased significantly in all categories of animals receiving 50 to $150 \mathrm{U}$ per day (Table 1). The storage (liver) and functional (plasma) iron pools were not modified with 0 or $10 \mathrm{U}$ rHuEpo, but when modified with higher doses of rHuEpo, were significantly depleted compared with baseline (Table 1). With 50 to $150 \mathrm{U}$ rHuEpo, normal rats showed depletion of the functional as well as storage pools consistent with iron deficiency, supplemented rats had functional iron deficiency but iron stores were equivalent to those of untreated normal rats, and overloaded rats retained adequate plasma iron levels in the presence of elevated stores.

In normal rats, treatment with rHuEpo produced dose-

Fig 3. Evolution of Hct, absolute reticulocyte count, and STfR during treatment with daily doses of $50 \mathrm{U}$ rHuEpo in nomal $(\Theta)$ or overloaded $(O)$ rats. Results are expressed as percentages of baseline value. (A) Hct; (B) absolute reticulocyte count; (C) sTfR.

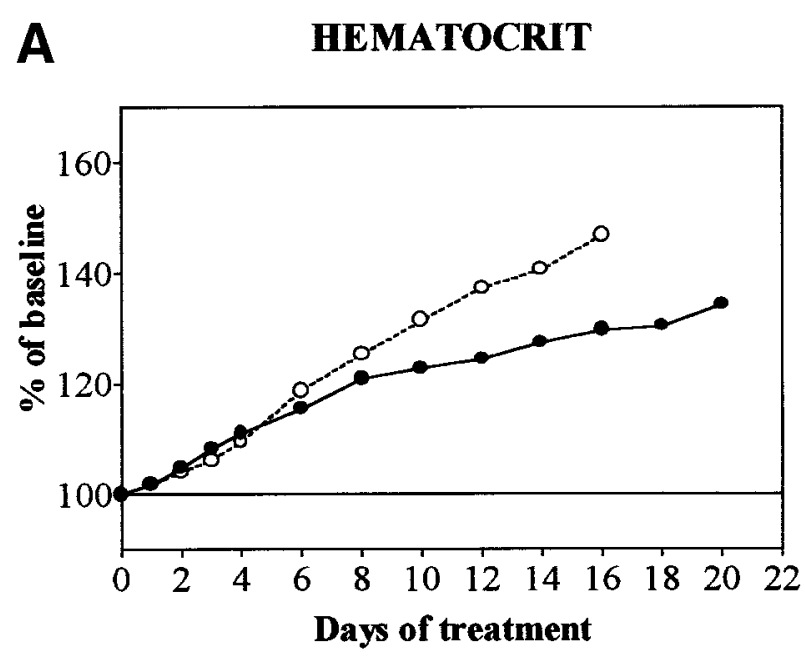

B RETICULOCYTES

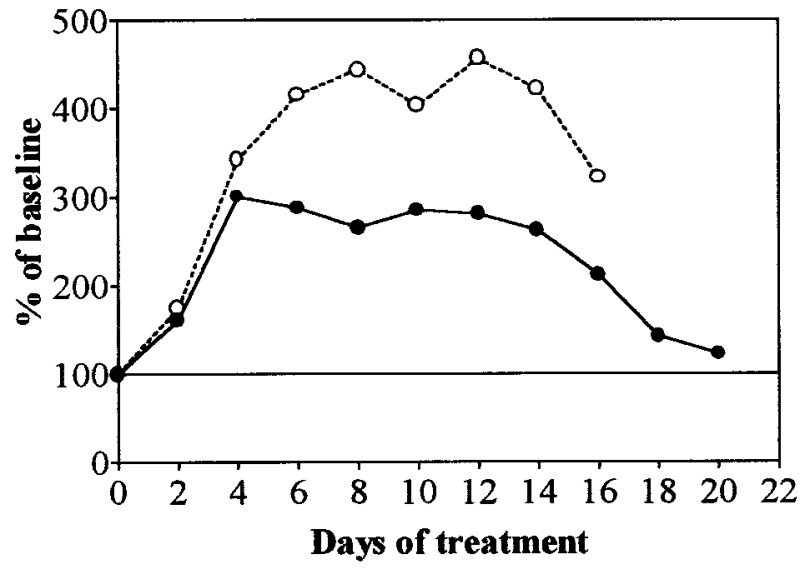

C sTfR

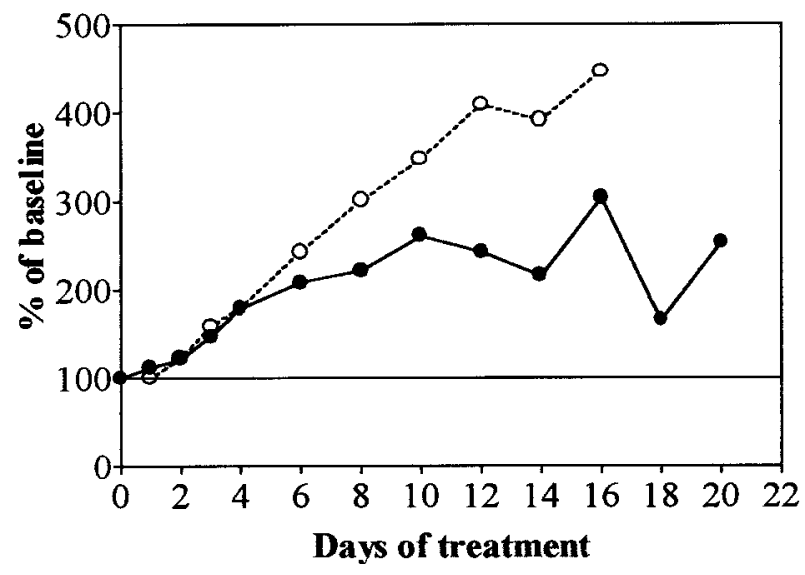


Table 1. Serum Iron, Liver Nonheme Iron, and Spleen Weight in Normal, Supplemented, and Overloaded Rats Treated for 0, 4, or 16 Days With 0, 10, 50, or 150 U rHuEpo Per Day

\begin{tabular}{|c|c|c|c|c|c|}
\hline Category & $\begin{array}{c}\text { Dose of rHuEpo } \\
(\mathrm{U} / \mathrm{d})\end{array}$ & $\begin{array}{l}\text { Duration of } \\
\text { Treatment (d) }\end{array}$ & $\begin{array}{c}\text { SeFe } \\
(\mu d / d L)\end{array}$ & $\begin{array}{l}\text { Liver Nonheme } \\
\quad \mathrm{Fe}(\mu \mathrm{g})\end{array}$ & $\begin{array}{l}\text { Spleen Weight } \\
(\mathrm{mg})\end{array}$ \\
\hline \multirow[t]{9}{*}{ Normal } & - & Baseline & $64 \pm 10$ & $326 \pm 156$ & $491 \pm 67$ \\
\hline & 0 & 4 & $73 \pm 16$ & $385 \pm 89$ & $527 \pm 75$ \\
\hline & & 16 & $54 \pm 15$ & $341 \pm 60$ & $524 \pm 48$ \\
\hline & 10 & 4 & $56 \pm 8$ & $312 \pm 51$ & $635 \pm 105$ \\
\hline & & 16 & $59 \pm 30$ & $318 \pm 114$ & $567 \pm 72$ \\
\hline & 50 & 4 & $25 \pm 12^{*}$ & $292 \pm 177$ & $845 \pm 167 \dagger$ \\
\hline & & 16 & $23 \pm 11^{*}$ & $131 \pm 33 \dagger$ & $861 \pm 148^{*}$ \\
\hline & 150 & 4 & $8 \pm 4 \ddagger$ & $267 \pm 106$ & $1,187 \pm 144 \ddagger$ \\
\hline & & 16 & $13 \pm 4 \ddagger$ & $115 \pm 9 \dagger$ & $1,105 \pm 117 \neq$ \\
\hline \multirow[t]{4}{*}{ Supplemented } & - & Baseline & - & - & - \\
\hline & 0 & 16 & $80 \pm 3$ & $1,042 \pm 92$ & $485 \pm 26$ \\
\hline & 50 & 16 & $64 \pm 24$ & $383 \pm 266 \dagger$ & $1,453 \pm 207 \S$ \\
\hline & 150 & 16 & $24 \pm 11 \S$ & $276 \pm 88 \ddagger$ & $1,508 \pm 207 \S$ \\
\hline \multirow[t]{5}{*}{ Overloaded } & - & Baseline & $132 \pm 26$ & $5,376 \pm 1,128$ & $687 \pm 83$ \\
\hline & 0 & 16 & $113 \pm 19$ & $4,203 \pm 1,602$ & $566 \pm 90$ \\
\hline & 10 & 16 & $76 \pm 11$ & $4,601 \pm 1,058$ & $676 \pm 23$ \\
\hline & 50 & 16 & $57 \pm 10^{*}$ & $2,333 \pm 942 \S$ & $1,103 \pm 118^{*}$ \\
\hline & 150 & 16 & $58 \pm 7 *$ & $2,282 \pm 1,710 \S$ & $2,003 \pm 571 \dagger$ \\
\hline
\end{tabular}

$P$ values are given for comparisons with rats of the same category in baseline conditions.

$* P<.001$.

$\dagger P<.05$.

$\ddagger P<.0001$

$\S P<.01$.

dependent effects on platelet counts (Fig 4A). With $10 \mathrm{U}$ rHuEpo per day, platelets remained stable and comparable to levels observed with $0 \mathrm{U}$. On the other hand, with $50 \mathrm{U}$ rHuEpo platelet counts increased to $120 \%$ of baseline after 4 days and remained stable thereafter. With $150 \mathrm{U}$ rHuEpo daily, they increased to $140 \%$ of baseline by day 8 to progressively level off at $120 \%$ at 14 to 20 days (Figs 4A and 5). In animals receiving $0 \mathrm{U}$ rHuEpo, supplemented or overloaded (Fig 4B) rats behave similarly to normal rats. Supplemented rats receiving 50 to $150 \mathrm{U}$ rHuEpo also increased platelets initially, but the peak was at day 4 , followed by a gradual return to baseline by day 10 and a moderate degree of thrombocytopenia later on (Fig 5). Overloaded rats receiving 50 to $150 \mathrm{U}$ rHuEpo also increased platelets at day 4. However, the duration of platelet increase was shorter and the importance of secondary thrombocytopenia was more pronounced (Figs $4 \mathrm{~B}$ and 5). This occurred in proportion to the dose of rHuEpo and reached $60 \%$ of baseline with $150 \mathrm{U}$ rHuEpo per day for 20 days (Fig 4B).

In normal animals previously splenectomized, treatment with high doses of rHuEpo was associated with a transient elevation of platelet counts peaking at day 4 before progressively returning to baseline, whereas unmanipulated animals maintained increased platelet counts throughout the experiment (Fig 6A). This contrasted with supplemented or overloaded rats (Fig 6B), in which unmanipulated, sham-operated, or splenectomized animals behave similarly, ie, first increased platelet counts and then developed secondary thrombocytopenia with high doses of rHuEpo.

Treatment with $150 \mathrm{U}$ rHuEpo was stopped after 20 days and rats were followed-up for 30 days after cessation of therapy (Fig 7). The Hct, reticulocytes, and sTfR levels decreased progressively, whereas platelet counts recovered in parallel over a period of 4 weeks. The evolution was identical in unmanipulated, sham-operated, or splenectomized animals.

\section{DISCUSSION}

In the present study, animals receiving $10 \mathrm{U}$ rHuEpo did not experience changes in platelet counts but had some increase in Hct and reticulocytes. However, as this happened without mobilization of storage iron and without increase in sTfR levels (the best quantitative measure of total erythropoiesis), this was probably achieved through shift reticulocytosis rather than true stimulation of erythropoietic activity. Rats treated with higher doses of rHuEpo underwent a true expansion of erythropoiesis, translating into progressive elevation of all erythroid parameters. Normal animals soon became limited in their capacity to respond because of the occurrence of iron deficiency. The augmentation of erythropoietic activity continued further in overloaded rats and these animals attained much higher sTfR values. Soluble TfR levels, thought in some situations to be a marker of (functional) iron deficiency, ${ }^{24}$ are therefore much more sensitive to changes in total erythropoietic activity than to changes in iron availability. This is consistent with our previous finding that patients with functional iron deficiency before treatment did not increase sTfR levels in response to rHuEpo. ${ }^{29}$

Our results confirm that high doses of rHuEpo induce significant changes in platelet counts. The platelet response appeared to undergo two different phases, ie, an initial moderate thrombocytosis and a secondary more pronounced thrombocytopenia. This diphasic pattern of response has already been described in animal models of hypoxia ${ }^{30-33}$ but not in models of rHuEpo therapy.

The initial increment in platelet counts was initiated within 48 hours of starting rHuEpo therapy and developed in a 
A

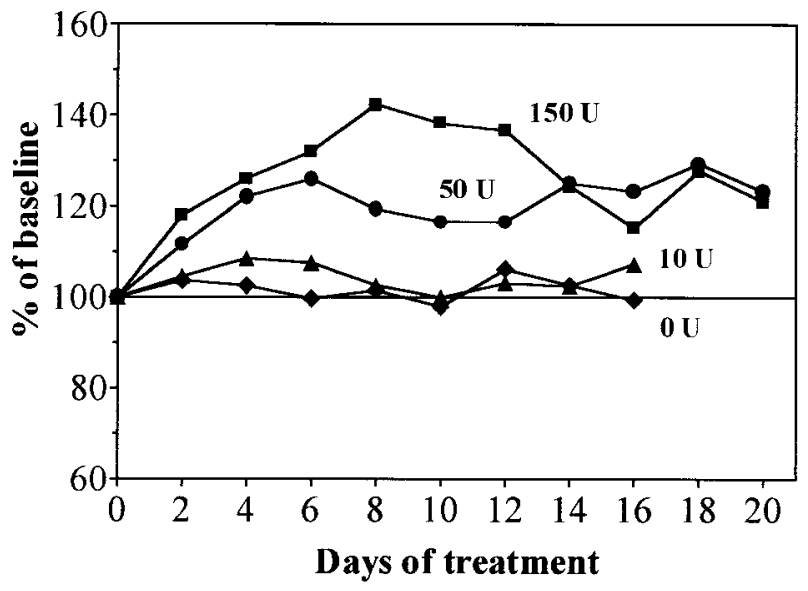

B Platelets

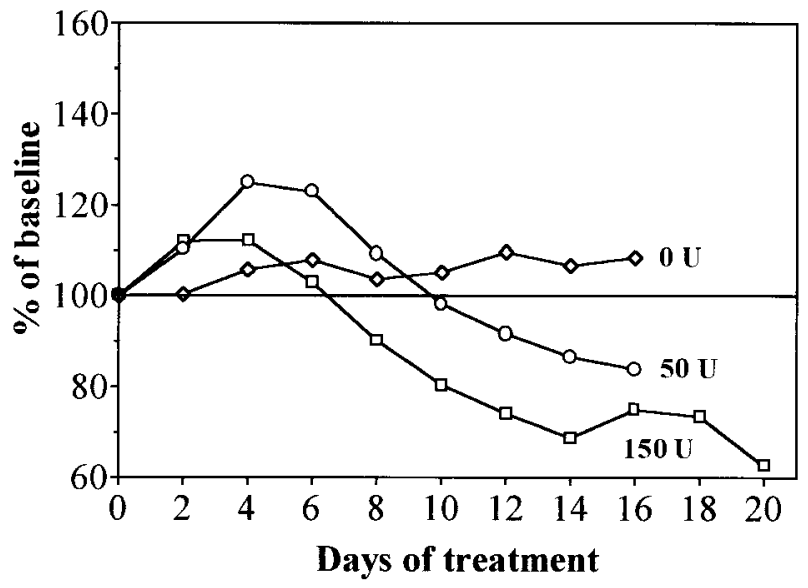

Fig 4. Evolution of platelet counts during treatment with daily doses of $\mathbf{0}$ (diamonds), 10 (triangles), 50 (circles), or 150 (squares) $\mathrm{U}$ rHuEpo. Results are expressed as percentages of baseline value. (A) Normal rats (solid symbols); (B) overloaded rats (open symbols).

dose-dependent fashion. This short-term elevation of platelet counts could be accounted for by several mechanisms, including changes in plasma volume or platelet survival and stimulation of megakaryocyte activity or global marrow function. Proportional shrinkage of plasma volume occurs only after rHuEpo treatment has produced changes in the red blood cell (RBC) mass. ${ }^{34}$ Furthermore, simultaneous elevations of RBCs and platelets should then be observed, but this was not the case in renal failure patients. ${ }^{2}$ An effect of rHuEpo on platelet survival has never been demonstrated, and there is no reason why this would be limited to patients in whom RBC production is adequately stimulated. ${ }^{2}$ A number of in vitro data support the concept that Epo directly stimulates megakaryopoiesis. Megakaryocytes have been shown to express constitutive ${ }^{35}$ or inducible ${ }^{36,37}$ high-affinity binding sites for Epo, resulting in enhanced growth in the presence of Epo. ${ }^{38}$ In vitro studies in humans as well as in mice have demonstrated that Epo promotes megakaryocytic colony formation and increases the size, ploidy, and number of megakaryocytes, as well as their DNA and protein synthesis and cytoplasmic process formation. ${ }^{38-42}$ Instead of directly stimulating megakaryocytes, Epo could also promote platelet production indirectly by enhancing global hematopoietic activity. Treatment of patients with end-stage renal failure rapidly resulted in an increase of not only erythroid progenitors, but also of colony-forming units megakaryocytes (CFU-Meg) and colony-forming unit-granulocyte-macrophage (CFU-GM) ${ }^{43}$ In such patients, platelet increments correlated with the degree of expansion of erythropoietic activity and platelet counts did not change in nonresponders until the Epo dose was increased and erythropoiesis begun to expand. ${ }^{2}$ In chronic liver disease, rHuEpo therapy ameliorates platelet counts in patients who also show a response of the erythroid lineage. ${ }^{44}$ In mice, elevated platelet counts correlated with increased Hct after 5 days of rHuEpo therapy. ${ }^{7}$ These observations suggest that the Epo effect on platelet formation parallels its effect on RBC production.

In animals with adequate iron supply, the initial elevation of platelet counts rapidly gave way to a substantial degree of thrombocytopenia. This also developed in a dose-dependent manner, with the decrease occurring earlier and being more pronounced in animals receiving higher doses of rHuEpo. In previous studies with rHuEpo, the initial elevation of platelet counts was followed by a return of platelets to control levels after 7 or 15 days. ${ }^{6,7}$ Actually, large, chronic doses of rHuEpo caused thrombocytopenia, decreased seleno-methionine incorporation into platelets, and reduced number of megakaryocytes in normal mice. ${ }^{11}$ Transgenic mice expressing the human Epo gene developed both polycythemia and a moderate degree of thrombocytopenia. ${ }^{10}$ Similarly, chronic hypoxia decreased platelet production, ${ }^{30-33,45}$ and this was shown to result from decreased differentiation of hematopoietic precursors into the megakaryocytic lineage. ${ }^{31,46}$ Increased erythropoiesis and not elevated RBCs (as produced by transfusion) was required for the thrombocytopenia to occur. ${ }^{33,47}$ Conversely, acute thrombo-

\section{PLATELETS}

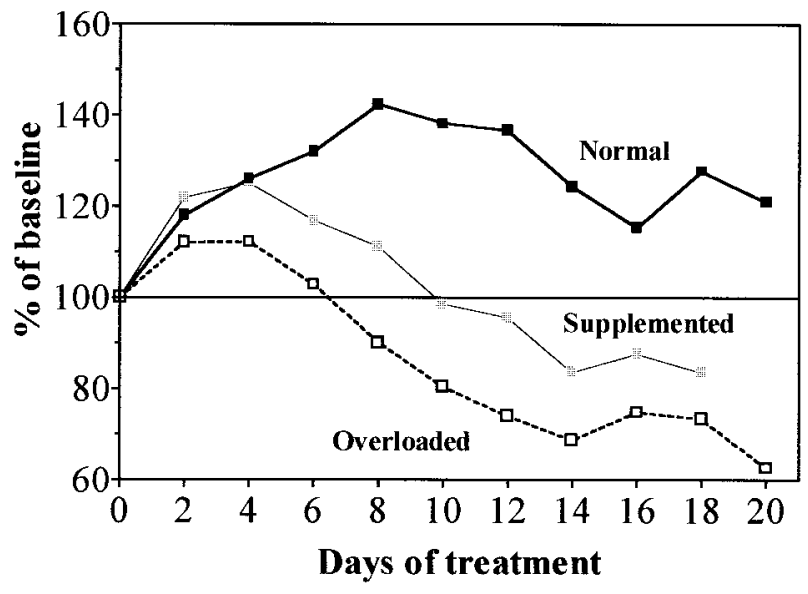

Fig 5. Evolution of platelet counts during treatment with daily doses of $150 \mathrm{U}$ rHuEpo in normal $(\square)$, supplemented $(\square)$, or overloaded ( $\square$ ) rats. Results are expressed as percentages of baseline value. 


\section{A PLATELETS}

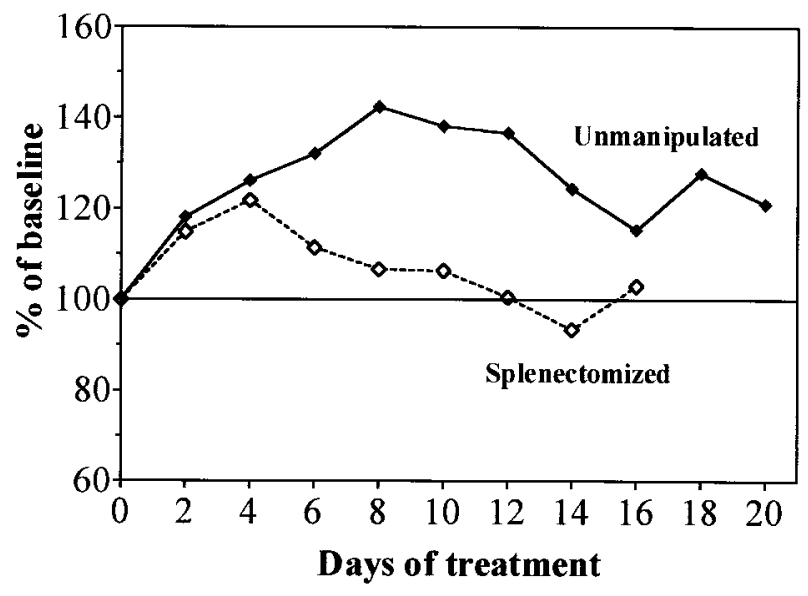

B PLATELETS

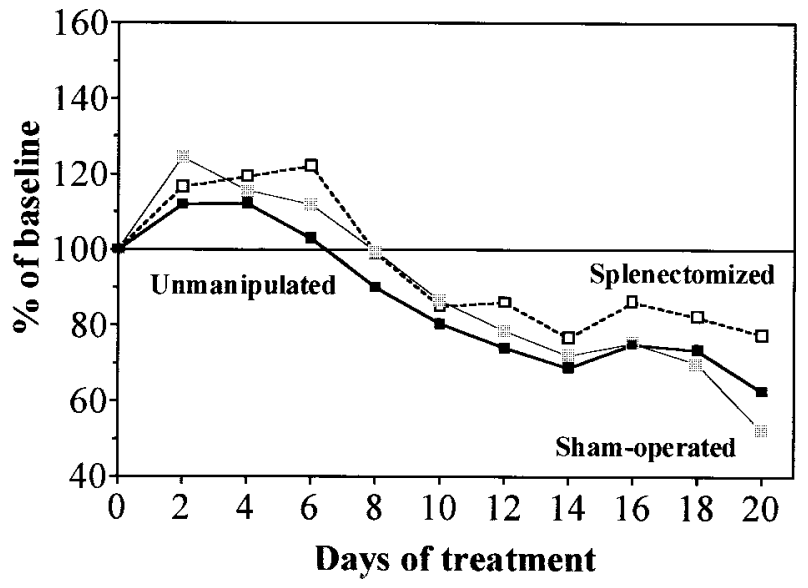

Fig 6. Evolution of platelet counts during treatment with daily doses of $150 \mathrm{U}$ rHuEpo in unmanipulated (solid symbols), shamoperated (shaded symbols), or splenectomized (open symbols) rats. Results are expressed as percentages of baseline value. (A) Normal rats (diamonds); (B) overloaded rats (squares).

cytopenia caused decreased erythropoiesis in some studies. ${ }^{11}$ Short-term thrombopoietin (Tpo) treatment in normal mice ${ }^{48}$ as well as chronic exposure to Tpo after gene transfer ${ }^{49}$ induced erythroid hypoplasia. However, this was at least partially explained by the development of myelofibrosis. Finally, Epoinduced thrombocytopenia is very unlikely to be due to increased platelet destruction, as observed with other growth factors such as macrophage colony-stimulating factor (MCSF).$^{50}$ During Epo-induced thrombocytopenia, there is substantial suppression of megakaryopoiesis ${ }^{11,31,46}$ and no change in platelet survival. ${ }^{30}$ Furthermore, recovery of a normal platelet count took several weeks after cessation of rHuEpo therapy, a finding difficult to reconcile with recovery after ending activation of the reticulo-endothelial system.

All this is suggestive of a pattern of reciprocal changes in erythropoiesis and megakaryopoiesis. Therefore, stem cell competition between erythroid and platelet precursors has been suggested to occur in these situations of prolonged, intense stimulation. ${ }^{11}$ Recent data support the concept that megakaryocytic and erythrocytic cell lineages share a common precursor. ${ }^{51}$ Multipotential $^{37}$ or bipotential ${ }^{52-54}$ cell lines expressing Epo receptors ${ }^{37,53}$ have been obtained in which erythroid differentiation can be induced through the action of Epo ${ }^{37,52}$ and megakaryocytic differentiation by Tpo. ${ }^{52,54}$ Such a bipotent erythromegakaryocytic progenitor has also been characterized in normal human bone marrow, ${ }^{55}$ coexpressing glycophorin A and glycoprotein IIIa. ${ }^{56}$

The effect of rHuEpo on platelet counts was strongly modulated by the iron status of the animals. Thrombocytopenia occurred earlier and was more pronounced in overloaded rats (no iron deficiency) than in supplemented animals (functional iron deficiency). Normal animals developed iron deficiency with depletion of iron stores that resulted in apparent protection from Epo-induced thrombocytopenia. Iron deficiency has been shown to be associated with thrombocytosis, ${ }^{13-17}$ but platelet counts tend to normalize $\mathrm{e}^{19,20,57}$ and thrombocytopenia can even occur ${ }^{18,58,59}$ when iron deficiency becomes very severe. This is consistent with the diphasic pattern of majored stimulation by endogenous Epo. Iron therapy ensures correction of platelet counts in infants ${ }^{18}$ or in rats ${ }^{19,20}$ with moderate iron deficiency, but has little effect in rats with severe iron deficiency anemia and normal platelet counts. ${ }^{19,20}$ In cases with very severe iron deficiency, iron therapy may even be associated with thrombocytopenia. ${ }^{60}$ Therefore, the effects of iron status and iron therapy on platelet production depend on the severity of iron deficiency.

However, changes in platelet counts during iron deficiency anemia could represent in part an effect of increased endogenous Epo stimulation in response to the anemia rather than an effect of iron deficiency per se. With moderate iron deficiency, a modest increase in serum Epo would stimulate platelet production, whereas with more severe anemia a major Epo response would engender thrombocytopenia. Accordingly, transfusion of RBCs into iron-deficient rats decreases platelet production, whereas transfusion into normal animals has no effect. ${ }^{20}$ In patients with renal failure receiving moderate doses of rHuEpo,

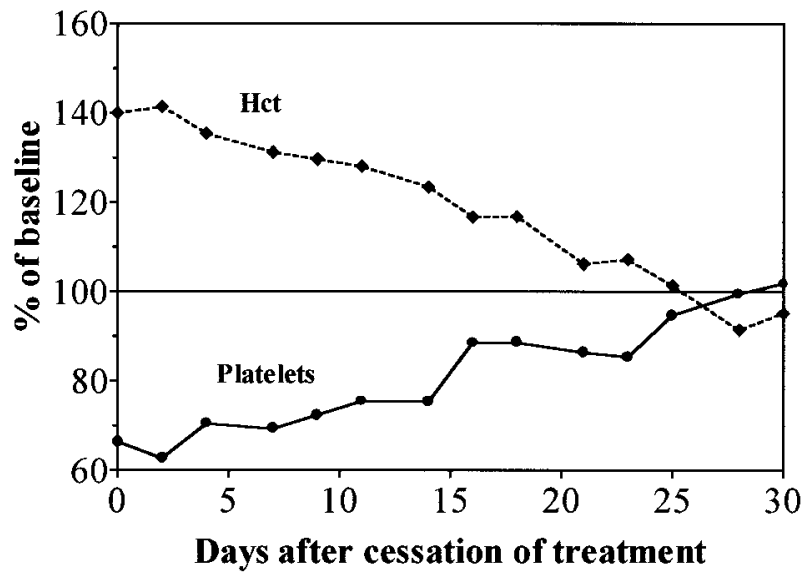

Fig 7. Evolution of Hct (---) and platelet counts (-) after cessation of treatment with daily doses of $\mathbf{1 5 0} \mathrm{U}$ rHuEpo in overloaded rats. Results are expressed as percentages of baseline value. 
relative platelet increments over baseline correlated inversely with relative changes of serum iron or transferrin saturation (an indication of erythroid marrow activity) rather than with absolute serum iron and transferrin saturation values (an indication of functional iron deficiency) or with ferritin levels (an indication of iron stores), emphasizing the role of marrow response to $\mathrm{rHuEpo}$ rather than that of iron deficiency alone. ${ }^{2}$ Our results in overloaded animals also show that the early stimulatory effect of rHuEpo on platelet production is a direct effect of treatment rather than an indirect effect of iron deficiency. Nevertheless, our data also show that with high doses of rHuEpo thrombocytopenia develops in inverse relationship to the degree of iron deficiency. This indicates that iron deficiency per se plays an important protective role against thrombocytopenia induced by high doses of rHuEpo in the presence of adequate iron supply.

Normal splenectomized animals showed a much less sustained platelet response to rHuEpo therapy compared with unmanipulated rats, but this discrepancy was no longer apparent in supplemented or overloaded rats (Fig 6). Previous studies in mice have shown that the stimulation effect of rHuEpo on megakaryocyte and CFU-Meg numbers may be limited to the spleen in intact mice and become significant in the bone marrow only after splenectomy. ${ }^{7,21,22}$ Splenectomized animals may thus be unable to maintain the positive response to rHuEpo observed in intact animals. It could also be possible that these rats experienced a higher degree of marrow erythroid expansion compensating for the absence of the spleen, thereby creating more competition with platelet production. However, this was no longer true in supplemented or overloaded rats. Splenic hematopoiesis may be less sensitive to Epo-induced competition, explaining why hypoxia is associated with a normal absolute number of megakaryocytes in the spleen ${ }^{61,62}$ and why splenectomy causes a further degree of hypoxia-induced thrombocytopenia in hypertransfused mice. ${ }^{63}$

A question remaining to be examined in further studies is whether the effects of rHuEpo on platelet production are a result of the dose itself or of the magnitude of the erythropoietic effect of that dose. For example, could a lower dose administered in a patient with decreased marrow function (because his number of hematopoietic progenitors is decreased by postchemotherapy stem cell damage or in the context of transplantation) bring about the same biological effects as those induced by higher doses of rHuEpo in the presence of a normal marrow function?

\section{REFERENCES}

1. Eschbach JW, Abdulhadi MH, Browne JK, Delano BG, Downing MR, Egrie JC, Evans RW, Friedman EA, Graber SE, Haley NR, Korbet S, Krantz SB, Lundin AP, Nissenson AR, Ogden DA, Paganini EP, Rader B, Rutsky EA, Stivelman J, Stone WJ, Teschan P, Van Stone JC, Van Wijk DB, Zuckerman K, Adamson JW: Recombinant human erythropoietin in anemic patients with end-stage renal disease. Results of a phase III multicenter clinical trial. Ann Intern Med 111:992, 1989

2. Beguin Y, Loo M, R'Zik S, Sautois B, Lejeune F, Rorive G, Fillet G: Effect of recombinant human erythropoietin on platelets in patients with anemia of renal failure: Correlation of platelet count with erythropoietic activity and iron parameters. Eur J Haematol 53:265, 1994

3. Sowade O, Ziemer S, Sowade B, Franke W, Messinger D, Ziebell E, Scigalla $\mathrm{P}$, Warnke H: The effect of preoperative recombinant human erythropoietin therapy on platelets and hemostasis in patients undergoing cardiac surgery. J Lab Clin Med 129:376, 1997

4. George JW, Bracco CA, Shannon KM, Davis GJ, Smith IL, Phibbs RH, Hendrickx AG: Age-related differences in erythropoietic response to recombinant human erythropoietin: Comparison in adult and infant rhesus monkeys. Pediatr Res 28:567, 1990

5. McDonald TP, Cottrell MB, Clift RE, Cullen WC, Lin FK: High doses of recombinant erythropoietin stimulate platelet production in mice. Exp Hematol 15:719, 1987

6. Berridge MV, Fraser JK, Carter JM, Lin FK: Effects of recombinant human erythropoietin on megakaryocytes and on platelet production in the rat. Blood 72:970, 1988

7. Shikama Y, Ishibashi T, Kimura H, Kawaguchi M, Uchida T, Maruyama Y: Transient effect of erythropoietin on thrombocytopoiesis in vivo in mice. Exp Hematol 20:216, 1992

8. Yonemura Y, Kawakita M, Fujimoto K, Sakaguchi M, Kusuyama T, Hirose J, Kato K, Takatsuki K: Effects of short-term administration of recombinant human erythropoietin on rat megakaryopoiesis. Int J Cell Cloning 10:18, 1992

9. Wolf RF, Peng J, Friese P, Gilmore LS, Burstein SA, Dale GL: Erythropoietin administration increases production and reactivity of platelets in dogs. Thromb Haemost 78:1505, 1997

10. Semenza GL, Traystman MD, Gearhart JD, Antonarakis SE: Polycythemia in transgenic mice expressing the human erythropoietin gene. Proc Natl Acad Sci USA 86:2301, 1989

11. McDonald TP, Clift RE, Cottrell MB: Large, chronic doses of erythropoietin cause thrombocytopenia in mice. Blood 80:352, 1992

12. Beguin Y: Prediction of response to optimize outcome of treatment with erythropoietin. Semin Oncol 25:27, 1998 (suppl 7)

13. Schloesser LL, Kipp MA, Wenzel FJ: Thrombocytosis in irondeficiency anemia. J Lab Clin Med 66:107, 1965

14. Harker LA, Finch CA: Thrombokinetics in man. J Clin Invest 48:963, 1969

15. Holter PH, Refsum HE: Postnatal anemia and thrombocytosis in suckling rabbits: Influence of delayed weaning and iron supplies. Pediatr Hematol Oncol 5:157, 1988

16. Dincol K, Aksoy M: On the platelet levels in chronic iron deficiency anemia. Acta Haematol 41:135, 1969

17. Kasper CK, Whissell YE, Wallerstein RO: Clinical aspects of iron deficiency. JAMA 191:359, 1965

18. Gross S, Keefer V, Newman AJ: The platelets in iron-deficiency anemia. I. The response to oral and parenteral iron. Pediatrics 34:315, 1964

19. Choi SI, Simone JV, Jackson CW: Megakaryocytopoiesis in experimental iron deficiency anemia. Blood 43:111, 1974

20. Choi SI, Simone JV: Platelet production in experimental iron deficiency anemia. Blood 42:219, 1973

21. Tsukada J, Misago M, Kikuchi M, Sato T, Ogawa R, Oda S, Chiba S, Eto S: The effect of high doses of recombinant human erythropoietin on megakaryocytopoiesis and platelet production in splenectomized mice. Br J Haematol 76:260, 1990

22. Grossi A, Vannucchi AM, Rafanelli D, Ferrini PR: Recombinant human erythropoietin has little influence on megakaryocytopoiesis in mice. Br J Haematol 71:463, 1989

23. Lee LG, Chen CH, Chiu LA: Thiazole orange: A new dye for reticulocyte analysis. Cytometry 7:508, 1986

24. Beguin Y, Huebers HA, Josephson B, Finch CA: Transferrin receptors in rat plasma. Proc Natl Acad Sci USA 85:637, 1988

25. Beguin Y, Clemons G, Pootrakul P, Fillet G: Quantitative assessment of erythropoiesis and functional classification of anemia based on measurements of serum transferrin receptor and erythropoietin. Blood 81:1067, 1993

26. Kaldor I: Studies on intermediary iron metabolism V. The measurement of non-haemoglobin tissue iron. Aust J Exp Biol Med Sci 32:795, 1954 
27. Johansson TB, Akselsson R, Johansson SAE: Elemental trace element analysis at the 10-12 level. Nucl Instrum Methods 84:141, 1970

28. Revised recommendations for the measurements of the serum iron in human blood. Iron Panel of the International Committee for Standardization in Haematology. Br J Haematol 75:615, 1990

29. Beguin Y, Loo M, R'Zik S, Sautois B, Lejeune F, Rorive G, Fillet $\mathrm{G}$ : Early prediction of response to recombinant human erythropoietin in patients with the anemia of renal failure by serum transferrin receptor and fibrinogen. Blood 82:2010, 1993

30. Jackson CW, Edwards CC: Biphasic thrombopoietic response to severe hypobaric hypoxia. Br J Haematol 35:233, 1977

31. McDonald TP, Cottrell MB, Steward SA, Clift RE, Swearingen CJ, Jackson CW: Comparison of platelet production in two strains of mice with different modal megakaryocyte DNA ploidies after exposure to hypoxia. Exp Hematol 20:51, 1992

32. McDonald TP, Cottrell MB, Clift R: Effects of short-term hypoxia on platelet counts in mice. Blood 51:165, 1978

33. McDonald TP: Platelet production in hypoxic and RBCtransfused mice. Scand J Haematol 20:213, 1978

34. Hughes RT, Cotes PM, Pippard MJ, Stevens JM, Oliver DO, Winearls CG, Royston JP: Subcutaneous administration of recombinant human erythropoietin to subjects on continuous ambulatory peritoneal dialysis: An erythrokinetic assessment. Br J Haematol 75:268, 1990

35. Fraser JK, Tan AS, Lin FK, Berridge MV: Expression of specific high-affinity binding sites for erythropoietin on rat and mouse megakaryocytes. Exp Hematol 17:10, 1989

36. Yoshida T, Ishida Y, Sasaki H, Inoue T, Kaku K, Kaneko T: Expression of high affinity binding sites for erythropoietin on L8057 cells, a mouse megakaryoblastic cell line, associated with cell differentiation. Am J Hematol 39:32, 1992

37. Hermine O, Mayeux P, Titeux M, Mitjavila MT, Casadevall N, Guichard J, Komatsu N, Suda T, Miura Y, Vainchenker W: Granulocytemacrophage colony-stimulating factor and erythropoietin act competitively to induce two different programs of differentiation in the human pluripotent cell line UT-7. Blood 80:3060, 1992

38. Sasaki H, Hirabayashi Y, Ishibashi T, Inoue T, Matsuda M, Kai S, Ikuta K, Yokoyama K, Yokota T, Maruyama Y, Natsuyama S: Effects of erythropoietin, IL-3, IL-6 and LIF on a murine megakaryoblastic cell line: Growth enhancement and expression of receptor mRNAs. Leuk Res 19:95, 1995

39. Ishibashi T, Koziol JA, Burstein SA: Human recombinant erythropoietin promotes differentiation of murine megakaryocytes in vitro. J Clin Invest 79:286, 1987

40. Tsukada J, Misago M, Kikuchi M, Sato T, Ogawa R, Ota T, Oda $\mathrm{S}$, Morimoto I, Chiba S, Eto S: Interactions between recombinant human erythropoietin and serum factor(s) on murine megakaryocyte colony formation. Blood 80:37, 1992

41 .An E, Ogata K, Kuriya S, Nomura T: Interleukin-6 and erythropoietin act as direct potentiators and inducers of in vitro cytoplasmic process formation on purified mouse megakaryocytes. Exp Hematol 22:149, 1994

42. Ishida Y, Yano S, Yoshida T, Tanaka H, Yamada Y, Kawano M, Kaneko T, Matsumoto N: Biological effects of recombinant erythropoietin, granulocyte-macrophage colony-stimulating factor, interleukin 3, and interleukin 6 on purified rat megakaryocytes. Exp Hematol 19:608, 1991

43. Dessypris E, Graber SE, Krantz SB, Stone WJ: Effects of recombinant erythropoietin on the concentration and cycling status of human marrow hematopoietic progenitor cells in vivo. Blood 72:2060, 1988
44. Pirisi M, Fabris C, Soardo G, Cecchin E, Toniutto P, Bartoli E: Thrombocytopenia of chronic liver disease corrected by erythropoietin treatment. J Hepatol 21:376, 1994

45. McDonald TP, Cottrell MB, Clift RE, Jackson CW: Effects of hypoxia on megakaryocyte size and number of $\mathrm{C} 3 \mathrm{H}$ and $\mathrm{BALB} / \mathrm{c}$ mice. Proc Soc Exp Biol Med 199:287, 1992

46. Rolovic Z, Basara N, Biljanovic Paunovic L, Stojanovic N, Suvajdzic N, Pavlovic Kentera V: Megakaryocytopoiesis in experimentally induced chronic normobaric hypoxia. Exp Hematol 18:190, 1990

47. Shaikh BS, Erslev AJ: Thrombocytopenia in polycythemic mice. J Lab Clin Med 92:765, 1978

48. Ulich TR, del Castillo J, Senaldi G, Kinstler O, Yin S, Kaufman S, Tarpley J, Choi E, Kirley T, Hunt P, Sheridan WP: Systemic hematologic effects of PEG-rHuMGDF-induced megakaryocyte hyperplasia in mice. Blood 87:5006, 1996

49. Yan XQ, Lacey D, Fletcher F, Hartley C, McElroy P, Sun Y, Xia M, Mu S, Saris C, Hill D: Chronic exposure to retroviral vector encoded mgdf (mpl-ligand) induces lineage-specific growth and differentiation of megakaryocytes in mice. Blood 86:4025, 1995

50. Baker GR, Levin J: Transient thrombocytopenia produced by administration of macrophage colony-stimulating factor: Investigations of the mechanism. Blood 91:89, 1998

51. McDonald TP, Sullivan PS: Megakaryocytic and erythrocytic cell lines share a common precursor cell. Exp Hematol 21:1316, 1993

52. Morgan DA, Class R, Soslau G, Brodsky I: Cytokine-mediated erythroid maturation in megakaryoblastic human cell line HU-3. Exp Hematol 25:1378, 1997

53. Bonsi L, Grossi A, Strippoli P, Tumietto F, Tonelli R, Vannucchi AM, Ronchi A, Ottolenghi S, Visconti G, Avanzi GC, Pegoraro L, Bagnara GP: An erythroid and megakaryocytic common precursor cell line (B1647) expressing both c-mpl and erythropoietin receptor (Epo-R) proliferates and modifies globin chain synthesis in response to megakaryocyte growth and development factor (MGDF) but not to erythropoietin (Epo). Br J Haematol 98:549, 1997

54. Komatsu N, Kirito K, Shimizu R, Kunitama M, Yamada M, Uchida M, Takatoku M, Eguchi M, Miura Y: In vitro development of erythroid and megakaryocytic cells from a UT-7 subline, UT-7/GM. Blood 89:4021, 1997

55. Debili N, Coulombel L, Croisille L, Katz A, Guichard J, Breton-Gorius J, Vainchenker W: Characterization of a bipotent erythromegakaryocytic progenitor in human bone marrow. Blood 88:1284, 1996

56. Bellucci S, Han ZC, Pidard D, Caen JP: Identification of a normal human bone marrow cell population co-expressing megakaryocytic and erythroid markers in culture. Eur J Haematol 48:259, 1992

57. Dinçol K, Aksoy M: On the platelet levels in chronic iron deficiency anemia. Acta Haematol 41:135, 1969

58. Berger M, Brass LF: Severe thrombocytopenia in iron deficiency anemia. Am J Hematol 24:425, 1987

59. Lopas H, Rabiner SF: Thrombocytopenia associated with iron deficiency anemia. Clin Pediatr 5:609, 1966

60. Soff GA, Levin J: Thrombocytopenia associated with repletion of iron in iron-deficiency anemia. Am J Med Sci 295:35, 1988

61. Petursson SR, Chervenick PA: Effects of hypoxia on megakaryocytopoiesis and granulopoiesis. Eur J Haematol 39:267, 1987

62. Cullen WC, McDonald TP: Effects of isobaric hypoxia on murine medullary and splenic megakaryocytopoiesis. Exp Hematol 17:246, 1989

63. Birks JW, Klassen LW, Gurney CW: Hypoxia-induced thrombocytopenia in mice. J Lab Clin Med 86:230, 1975 